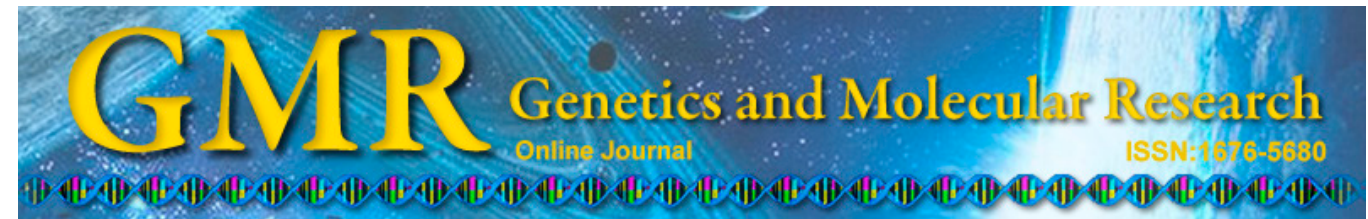

\title{
Expression of human amyloid precursor protein in Drosophila melanogaster nerve cells causes a decrease in presynaptic gene mRNA levels
}

\author{
D.I. Rodin ${ }^{1}$, A.L. Schwarzman ${ }^{1,2}$ and S.V. Sarantseva ${ }^{1}$ \\ ${ }^{1}$ Molecular and Radiation Biophysics Division, \\ National Research Centre "Kurchatov Institute" B.P. Konstantinov Petersburg \\ Nuclear Physics Institute, Gatchina, Russia \\ ${ }^{2}$ Molecular Genetics Department, \\ Institute of Experimental Medicine of The North-West Branch of the Russian \\ Academy of Medical Sciences, Saint-Petersburg, Russia
}

Corresponding author: D.I. Rodin

E-mail: rodin.dmitry@icloud.com

Genet. Mol. Res. 14 (3): 9225-9232 (2015)

Received December 3, 2014

Accepted May 26, 2015

Published August 10, 2015

DOI http://dx.doi.org/10.4238/2015.August.10.2

ABSTRACT. Amyloid precursor protein (APP) is a key player in Alzheimer's disease. The proteolytic cleavage of APP results in various short peptide fragments including the toxic amyloid-beta peptide, which is a main component of senile plaques. However, the functions of APP and its processed fragments are not yet well understood. Here, using real-time polymerase chain reaction, we demonstrate that exogenous expression of APP, its mutant form APP-Swedish, or two truncated forms in Drosophila melanogaster causes a significant $(\mathrm{P} \leq 0.05)$ drop in the mRNA levels of the presynaptic proteins synaptotagmin-1 and neuronal synaptobrevin. The results obtained from this study suggest a potential role of APP or its fragments in the regulation of synaptic gene transcription.

Key words: Alzheimer's disease; Drosophila; Synaptic protein; Amyloid precursor protein 


\section{INTRODUCTION}

Alzheimer's disease (AD) is a progressive neurodegenerative disorder that is characterized by loss of cognitive and functional abilities. One of the main pathomorphological markers of $\mathrm{AD}$ is extracellular senile plaques that generally consist of the amyloid-beta peptide $(\mathrm{A} \beta)$. To date there are several hypotheses to explain the development of AD but the most accepted is the amyloid cascade hypothesis (Haass and Selkoe, 2007; Walsh and Selkoe, 2007; Zhang et al., 2014).

According to this hypothesis, production of toxic $A \beta$ oligomers causes neurodegeneration and the development of AD. The primary evidence in support of this hypothesis is that familial forms of AD are caused by mutations in several genes that encode proteins involved in the generation of $\mathrm{A} \beta$, including the APP gene, and the presenilin 1 (PS1) and 2 (PS2) genes (Hardy and Selkoe, 2002; Haass and Selkoe, 2007). A $\beta$ oligomers are considered to be the main agents in the development of synapse dysfunction in the case of familial forms of AD (Walsh et al., 2002; Walsh and Selkoe, 2004). In contrast, an alternative hypothesis suggests that disruption of the normal cellular functions of APP is the main cause of synapse dysfunction in AD (Sarantseva et al., 2009; Nizzari et al., 2012).

It has been demonstrated that early memory and cognitive function impairment during the progression of AD strongly correlate with synapse dysfunction (Terry et al., 1991), accompanied by a considerable reduction of the levels of certain presynaptic proteins such as synaptophysin and synaptotagmin in the cortex and hippocampus of patients. Synapse dysfunction occurs well before $A \beta$ accumulation (Masliah and Mallory, 2001), but this effect has not yet been successfully explained.

In our previous study, we showed that hyper-expression of human APP in Drosophila melanogaster nerve cells led to neurodegeneration in fly brain, as well as loss of memory and learning ability, which are considered to be the processes observed in AD pathogenesis. Consequently, we assumed that hyper-expression of $A P P$ in the nerve cells of transgenic flies led to neuropathological processes specific for $\mathrm{AD}$. In the transgenic flies, we observed synapse disruption characterized by a reduced level of the proteins synaptotagmin 1 (syt1) and neuronal synaptobrevin (n-syb) in the mushroom body brain area of Drosophila (Sarantseva et al., 2009). However, the mechanism that was responsible for the synaptic protein level drop was not clear.

In this study, we show that hyper-expression of the $A P P$ gene and its fragments affect transcription of the presynaptic genes syt 1 and $n$-syb and that the decrease in presynaptic proteins is preceded by a drop in the corresponding gene expression levels.

\section{MATERIAL AND METHODS}

\section{Drosophila strains used for experiments}

Drosophila strains used in this study included $U A S-A P P$ (henceforth referred to as $A P P$ ) carrying the human $A P P$ gene, $U A S-A P P-S w e d i s h(A P P-S w)$ carrying the human $A P P$ gene with a mutation that leads to the familial form of $\mathrm{AD}, U A S-A P P \triangle N T$ (APP $\triangle N T)$ and $U A S-A P P \triangle C T(A P P \triangle C T)$, carrying the truncated forms of APP695, and UAS-BACE (BACE), carrying the human beta-secretase gene. All APP strains were obtained from the Drosophila Bloomington Stock Center (Indiana University, Bloomington, IN, USA); UAS-BACE was kindly provided by R. Reifegerste. $A P P$, its forms, and $B A C E$ were expressed in Drosophila 
neurons using the tissue-specific transcription driver elav-GAL4c155 (Indiana University, Bloomington, IN, USA).

Flies were kept on standard yeast medium at a temperature of $25^{\circ} \mathrm{C}$ and a photoperiod of $12 \mathrm{~h}$.

\section{RNA extraction and reverse transcription}

For each experiment, 40 flies were frozen in liquid nitrogen and decapitated. Heads were homogenized and RNA was extracted using a ZR Tissue \& Insect RNA MicroPrep ${ }^{\text {TM }}$ kit (Zymo Research, Irvine, CA, USA). During extraction, RNA samples were treated with DNase to reduce the amount of genomic DNA. RNA extraction was followed by reverse transcription with Oligo(dT) 18 Primers and RevertAid M-MuLV Reverse Transcriptase from the RevertAid ${ }^{\mathrm{TM}}$ First-Strand cDNA Synthesis Kit (Thermo Scientific, Waltham, MA, USA).

\section{Real-time polymerase chain reaction (PCR)}

The mRNA level for the syt 1 and $n$-syb genes were estimated by TaqMan real-time PCR with ABI PRISM 7000 Sequence Detection System (Applied Biosystems, Waltham, MA, USA). The RP-49 and GAPDH2 genes were used as the reference genes. Primer and probe sequences (Bigle, Russia) used for PCR were as follows: syt1: fwd 5'-CCT GGT CAG CGT TGA AGG A-3', rev 5'-GCA GCG AGA AGC AGA TAT CT-3', probe 5'-FAM-AGG GCG GAC AGG AAA-RTQ1-3'; n-syb: fwd 5'-GGC GGC GTG TAA GCA ATC-3', n-syb rev 5'CCC GCT GAA GGA GCA CAC TA-3', probe 5'-FAM-CGC TGC CAG GAC GAA AGT TTC TCG A-RTQ1-3'; PSN: fwd 5'-TGG ACT GCC TGG GCT GTA-3', rev 5'-TCC TCT TGG CGAAAG GAC A-3', probe 5'-FAM-CTG CCA TTT CTA TTT GGG ATC TT-RTQ1-3'; RP49: fwd 5'-AGC ACT TCA TCC GCC ACC-3', rev 5'-CGA CGC ACT CTG TTG TCG-3', probe 5'-R6G-CTA AGC TGT CGC ACA AAT GGC G-BHQ1-3'; and GAPDH2: fwd 5'-TGG CCAAGGTGATCAACGACAA-3', rev 5'-ACAACTTGCCGGAAGGTCCAT-3', probe 5'-R6G-TGA TGACCACCGTTCATGCCACCACCGCT-BHQ1-3'. Denaturation at $95^{\circ} \mathrm{C}$ for 10 min was followed by 50 amplification cycles (melting at $95^{\circ} \mathrm{C}$ for $30 \mathrm{~s}$; annealing at $60^{\circ} \mathrm{C}$ for $30 \mathrm{~s}$; synthesis at $72^{\circ} \mathrm{C}$ for $30 \mathrm{~s}$ ). The mRNA levels of $s y t 1$ and $n-s y b$ were normalized to mRNA levels of GAPDH 2 or RP-49. The relative amount of syt 1 and $n$-syb mRNA was estimated using relative standard curve method according to protocol provided by Applied Biosystems. Standard curves were built for genes of interest as well as for the reference genes. Every sample was tested in triplicate.

\section{Statistical analysis}

Statistical analysis was performed using the KyPlot software (KyensLab Inc., Tokyo, Japan). One-way ANOVA was followed by planned multiple comparisons between relevant groups with the Tukey-Kramer test.

\section{RESULTS}

We studied the effect of expression of $A P P$, its truncated forms, and $A P P-S w$ in nerve cells of Drosophila on the mRNA levels of the presynaptic proteins syt1 and n-syb. 
A $\beta$ production is a result of APP cleavage by $\beta$ - and $\gamma$-secretases. Drosophila has no or minimal activity of $\beta$-secretase and the Drosophila homologue of the human APP gene (APPL) does not have the A $\beta$ sequence (Luo et al., 1990; Fossgreen et al., 1998; Greeve et al., 2004). This allows us to analyze the effects of $A P P$ expression in Drosophila independently from $\mathrm{A} \beta$ effects.

Levels of syt 1 and $n$-syb mRNA were analyzed in 2- and 30-day-old flies of the following genotypes: elav/+ (control), elav; APP, elav; APP-Sw, elav; APP $\triangle C T$, elav; APP $\triangle N T$, elav; $A P P / B A C E$, and elav; $B A C E ; A P P-S w$. The results were normalized relatively to the control genotype, elav;+.

The mRNA levels of syt 1 and $n$-syb, normalized to the reference genes $R P-49$ and GAPDH2 in flies aged 2 and 30 days, are presented in Figures 1 and 2, respectively.

According to the data, the relative mRNA levels of $s y t 1$ and $n-s y b$ were decreased even during the first days of life in all transgenic flies analyzed. The expression level of syt 1 in 30-dayold flies was significantly reduced $(\mathrm{P} \leq 0.05)$ compared to control flies of the same age, with the exception of the single genotype, $A P P \triangle C T$, where statistical significance was not obtained, although a similar tendency toward reduction of expression level was observed. The expression level of $n$-syb in 30-day-old flies was reduced in flies expressing $A P P$ and $A P P-S w$.

Furthermore, in order to demonstrate an absence of an $A P P$ hyper-expression effect on the overall profile of organismal gene expression, we analyzed the expression level of Drosophila psn. The mRNA levels of $p s n$ in flies aged 2 and 30 days, normalized to the reference genes RP-49 and GAPDH2, are presented in Figure 3. No reduction of psn mRNA level was observed in any of the genotypes analyzed. In contrast, we observed increased transcriptional activity of $p s n$ in 30-day-old flies.

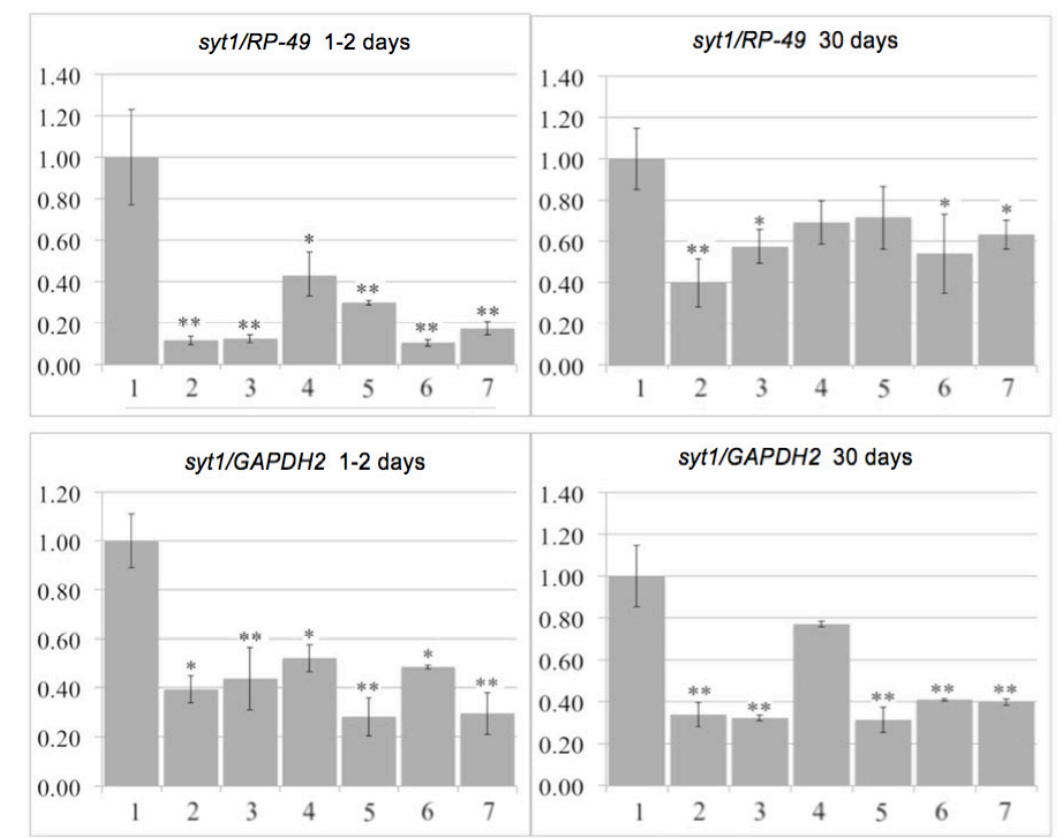

Figure 1. Relative mRNA expression levels of syt1 in 2- or 30-day-old flies. Genotypes: 1) elav (control); 2) elav; APP; 3) elav; APP-Sw; 4) elav; APP $\triangle \mathrm{CT}$; 5) elav; APP $\triangle \mathrm{NT}$; 6) elav; APP/BACE; 7) elav; BACE; APP-Sw. mRNA levels of syt 1 were normalized to the reference genes $R P-49$ (top) and $G A P D H 2$ (bottom). ${ }^{*} \mathrm{P} \leq 0.05, * * \mathrm{P} \leq 0.001$. 


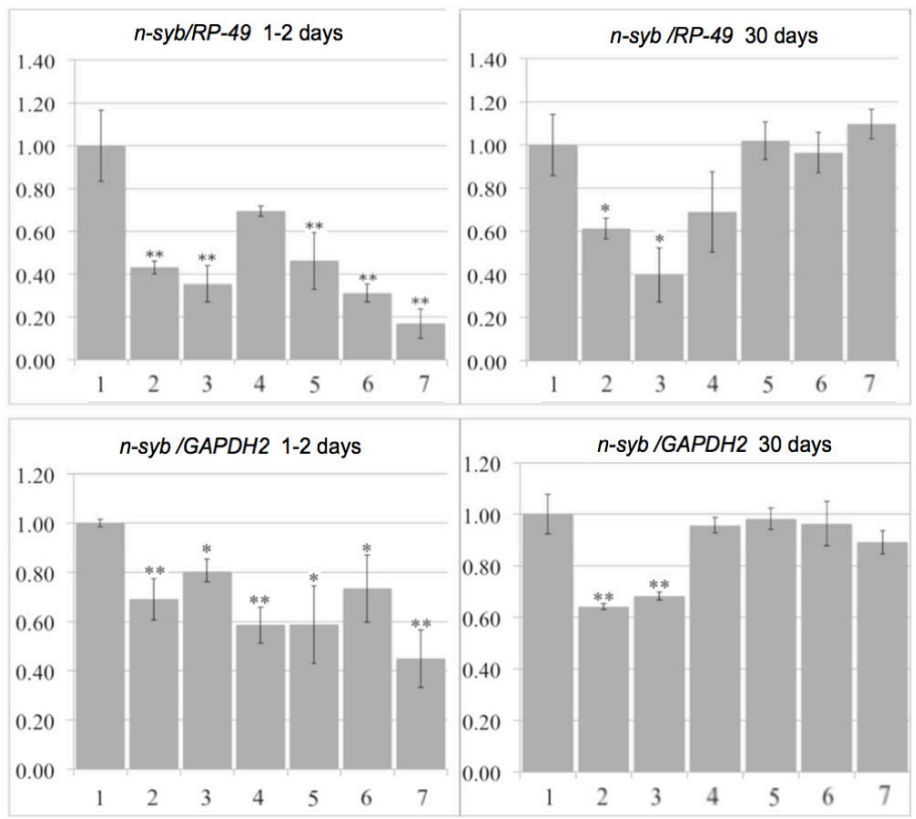

Figure 2. Relative mRNA expression levels of $n$-syb in 2- or 30-day-old flies. Genotypes: 1) elav (control); 2) elav; APP; 3) elav; APP-Sw; 4) elav; APP $\Delta$ CT; 5) elav; APP $\Delta$ NT; 6) elav; APP/BACE; 7) elav; BACE; APP-Sw. mRNA levels of $n$-syb were normalized to the reference genes $R P-49$ (top) and $G A P D H 2$ (bottom). ${ }^{*} \mathrm{P} \leq 0.05, * * \mathrm{P} \leq 0.001$.

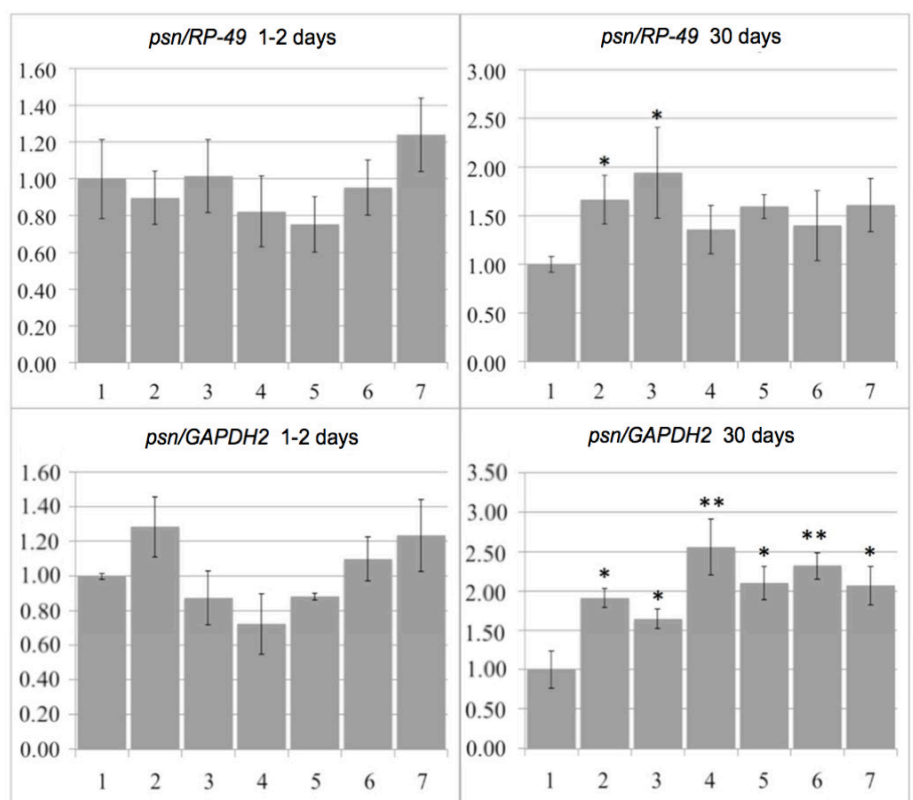

Figure 3. Relative mRNA expression level of $p s n$ in 2- or 30-day-old flies. Genotypes: 1) elav (control); 2) elav; APP; 3) elav; APP-Sw; 4) elav; APP $\triangle \mathrm{CT}$; 5) elav; APP $\triangle \mathrm{NT}$; 6) elav; APP/BACE; 7) elav; BACE; APP-Sw. mRNA levels of $p s n$ were normalized to the reference genes $R P-49$ (top) and GAPDH2 (bottom). ${ }^{*} \mathrm{P} \leq 0.05,{ }^{*} \mathrm{P} \leq 0.001$. 


\section{DISCUSSION}

Many studies show that early memory and cognitive function impairment in patients with Alzheimer's disease are followed by a considerable reduction of presynaptic protein levels in the brain (Masliah and Terry, 1993; Davidsson and Blennow, 1998). The level of synaptotagmin was considerably reduced in the areas responsible for memory formation and consolidation: hippocampus and entorhinal area of cortex, while at the same time the decrease in synaptobrevin level was independent from brain area (Sze et al., 2000). Similar results were observed in transgenic mice with hyper-expression of human APP (Chapman et al., 1999; Mucke et al., 2000). For example, a study of synaptic density in the brain of mice with hyper-expression of $A P P-S w$ showed a significant reduction of the level of syt protein in the cortex and hippocampus (Wang et al, 2012). Unfortunately, these effects have not yet been successfully and clearly interpreted. Transcript analysis carried out on the brains of patients with sporadic AD (average age of 82.7 years) using Affymetrix HuFL chips showed a reduced level of mRNA transcripts in most synaptic protein groups (Yao et al., 2003). On the other hand, experiments on transgenic mice ( 22 months old) showed a wide spectrum of synaptic protein levels (Yao et al., 2003). In addition, significant reductions of protein expression levels of VAMP1 and VAMP2 (coding 2 isoforms of synaptobrevin) and of syt (Mufson et al., 2002) were registered in the brains of patients with AD (Bossers et al., 2009). It should be noted that in the experiments described above there were no data for the early stages of $\mathrm{AD}$, which could potentially explain the inability of these studies to distinguish a primary effect of impaired synaptogenesis from other effects of the neuropathological process.

D. melanogaster, a genetically well studied model organism, has been widely used as an experimental model for studying mechanisms underlying neurodegenerative diseases including AD (Finelli et al., 2004; Greeve et al., 2004; Bilen and Bonini, 2005). It has been shown that the genes responsible for formation of the $\gamma$-secretase complex are highly conserved between Drosophila and mammals; accordingly, Drosophila has an active $\gamma$-secretase complex (Fossgreen et al., 1998). However, the Drosophila homolog of human $\beta$-secretase (dBACE) does not cleave APP at the $\beta$-site, and therefore dBACE cleavage of $A P P$ does not lead to A $\beta$ formation in flies (Greeve et al., 2004). In addition, whereas Drosophila has a homolog of human APP (APPL), the A $\beta$ sequence is not conserved in APPL (Rosen et al., 1989). However, it has been shown that expression of human APP in combination with the human $\beta$-secretase gene in Drosophila leads to $A \beta$ deposition and associated pathologies (Greeve et al., 2004).

Modeling AD in Drosophila allows estimation of the very early changes in synaptic protein levels and of alterations in cognitive function. In our previous studies, we demonstrated that hyper-expression of $A P P$ and $A P P-S w$ together with $B A C E$ co-expression caused a significant decrease in syt1 and n-syb protein levels in the antennal lobes and mushroom body, which are Drosophila brain structures responsible for memory formation. The decrease in protein levels correlated with disruption of cognitive function (odor recognition) (Sarantseva et al., 2009).

In our current research, we studied the effects of $A P P$ expression on the mRNA levels of synaptic proteins in transgenic Drosophila, which allowed for the discrimination of the effects of exogenous APP and A $\beta$. We demonstrated that $A P P$ hyper-expression changed the mRNA levels of $s y t 1$ and $n-s y b$. The data obtained in our experiments showed significant reduction of syt1 mRNA levels in 2-day-old flies in all genotypes tested, consequent to expression of $A P P$ and its variant forms as well as with co-expression of $A P P$ and $B A C E$, which leads to $\mathrm{A} \beta$ production. Similar results were observed in 30-day-old flies for syt1, with the exception of 
flies that expressed the shortened forms of $A P P: A P P \triangle C T$ and $A P P \triangle N T$. However, in 30-dayold flies the mRNA level of $n$-syb was reduced only in flies with full-length $A P P$ expression $(A P P$ and $A P P-S w)$. As previously described, the use of transgenic Drosophila allowed us to distinguish the effects of $A P P$ expression from those of $A \beta$ production. The results obtained in our experiments showed that the reduced mRNA levels of syt 1 and $n$-syb were caused by APP expression independently from $A \beta$ secretion. Although our study was carried out on transgenic flies, other studies have demonstrated that $A P P$ gene duplication caused AD development in five families with autosomal dominant early-onset Alzheimer disease (Rovelet-Lecrux et al., 2006).

We assume that not only A $\beta$ but also other fragments derived after APP processing might play an important role in the change of presynaptic gene expression levels. For example, it has been shown that the C-terminal fragment of APP-AICD, a 6-kDa protein cleaved from APP by $\gamma$-secretase, can penetrate the nucleus and bind with different intracellular adoptive proteins, influencing many cellular processes including gene expression, synaptic plasticity, and memory formation (Cao and Südhof, 2001; Gao and Pimplikar, 2001; Kim et al., 2003; Muller et al., 2007). On the other hand, APP itself is also able to participate in transcriptional regulation. Neuronal APP hyper-expression has been shown to reduce the mRNA levels of proteins involved in cholesterol metabolism: SREBP, HMGCR, and cholesterol 24-hydroxylase) (Pierrot et al., 2013).

In conclusion, transcriptional disturbance of the genes encoding synaptic proteins might underlie the alterations of synaptic plasticity in patients with $\mathrm{AD}$ and can be recognized as one of the early events of the disease. $A P P$ expression changes or $A P P$ mutations might lead to reduction of the mRNA levels of synaptic proteins and thereby cause synaptic pathology in patients with AD.

\section{ACKNOWLEDGMENTS}

Research supported by the Russian Foundation for Basic Research.

\section{REFERENCES}

Bilen N and Bonini NM (2005). Drosophila as a model for human neurodegenerative disease. Annu. Rev. Genet. 39: 153-171. Bossers K, Heetveld S, Swaab DF, Verhaagen J, et al. (2009). A meta-analysis of microarray-based gene expression studies in Alzheimer's disease. In: Spot the difference: microarray analysis of gene expression changes in Alzheimer's and Parkinson's Disease (Thesis). Royal Netherlands Academy of Arts and Sciences, Amsterdam, 135-153.

Cao X and Südhof TC (2001). A transcriptionally active complex of APP with Fe65 and histone acetyltransferase Tip60. Science 293: 115-120.

Chapman PF, White GL, Jones MW, Cooper-Blacketer D, et al. (1999). Impaired synaptic plasticity and learning in aged amyloid precursor protein transgenic mice. Nat. Neurosci. 2: 271-276.

Davidsson P and Blennow K (1998). Neurochemical dissection of synaptic pathology in Alzheimer's disease. Int. Psychogeriatr. 10: 11-23.

Finelli A, Kelkar A, Song H-J, Yang H, et al. (2004). A model for studying Alzheimer's AB42-induced toxicity in Drosophila melanogaster. Mol. Cell. Neurosci. 26: 365-375.

Fossgreen A, Brückner B, Czech C, Masters CL, et al. (1998). Transgenic Drosophila expressing human amyloid precursor protein show gamma-secretase activity and a blistered-wing phenotype. Proc. Natl. Acad. Sci. U.S.A. 95: 13703-13708.

Gao Y and Pimplikar SW (2001). The gamma-secretase-cleaved C-terminal fragment of amyloid precursor protein mediates signaling to the nucleus. Proc. Natl. Acad. Sci. U.S.A. 98: 14979-14984.

Greeve I, Kretzschmar D, Tschäpe JA, Beyn A, et al. (2004). Age-dependent neurodegeneration and Alzheimer-amyloid plaque formation in transgenic Drosophila. J. Neurosci. 24: 3899-3906.

Haass C and Selkoe DJ (2007). Soluble protein oligomers in neurodegeneration: lessons from the Alzheimer's amyloid beta-peptide. Nat. Rev. Mol. Cell Biol. 8: 101-112. 
Hardy J and Selkoe DJ (2002). The amyloid hypothesis of Alzheimer's disease: progress and problems on the road to therapeutics. Science 297: 353-356.

Kim HS, Kim EM, Lee JP, Park CH, et al. (2003). C-terminal fragments of amyloid precursor protein exert neurotoxicity by inducing glycogen synthase kinase-3beta expression. FASEB J. 17: 1951-1953.

Luo L, Martin-Morri LE and White K (1990). Identification, secretion, and neural expression of APPL Drosophila protein similar to human amyloid protein precursor. J. Neurosci. 10: 3849-3861.

Masliah E and Terry R (1993). The role of synaptic proteins in the pathogenesis of disorders of the central nervous system. Brain Pathol. 3: 77-85.

Masliah E and Mallory M (2001). Altered expression of synaptic proteins occurs early during progression of Alzheimer's disease. Neurology 56: 127-129.

Mucke L, Masliah E, Yu GQ, Mallory M, et al. (2000). High-level neuronal expression of a beta 1-42 in wild-type human amyloid protein precursor transgenic mice. Synaptotoxicity without plaque formation. J. Neurosci. 20: 4050-4058.

Mufson EJ, Counts SE and Ginsberg SD (2002). Gene expression profiles of cholinergic nucleus basalis neurons in Alzheimer's disease. Neurochem. Res. 27: 1035-1048.

Muller T, Concannon CG, Ward MW, Walsh CM, et al. (2007). Modulation of gene expression and cytoskeletal dynamics by the amyloid precursor protein intracellular domain (AICD). Mol. Biol. Cell 18: 201-210.

Nizzari M, Thellung S, Corsaro A, Villa V, et al. (2012). Neurodegeneration in Alzheimer disease: role of amyloid precursor protein and Presenilin 1 intracellular signaling. J. Toxicol. 2012: 187297.

Pierrot N, Tyteca D, D’Auria L, Dewachter I, et al. (2013). Amyloid precursor protein controls cholesterol turnover needed for neuronal activity. EMBO Mol. Med. 5: 608-625.

Rosen DR, Martin-Morris L, Luo LQ and White K (1989). A Drosophila gene encoding a protein resembling the human beta-amyloid protein precursor. Proc. Natl. Acad. Sci. U.S.A. 86: 2478-2482.

Rovelet-Lecrux A, Hannequin D, Raux G, Le Meur N, et al. (2006). APP locus duplication causes autosomal dominant early-onset Alzheimer disease with cerebral amyloid angiopathy. Nat. Genet. 38: 24-26.

Sarantseva S, Timoshenko S, Bolshakova O, Karaseva E, et al. (2009). Apolipoprotein E-mimetics inhibit neurodegeneration and restore cognitive functions in a transgenic Drosophila model of Alzheimer's disease. PLoS One 4: e8191.

Sze CI, Bi H, Kleinschmidt-DeMasters BK, Filley CM, et al. (2000). Selective regional loss of exocytotic presynaptic vesicle proteins in Alzheimer's disease brains. J. Neurol. Sci. 175: 81-90.

Terry RD, Masliah E, Salmon DP, Butters N, et al. (1991). Physical basis of cognitive alterations in Alzheimer's disease: Synapse loss is the major correlate of cognitive impairment. Ann. Neurol. 30: 572-580.

Walsh DM and Selkoe DJ (2004). Deciphering the molecular basis of memory failure in Alzheimer's disease. Neuron 44: 181-193.

Walsh DM and Selkoe DJ (2007). A beta oligomers - a decade of discovery. J. Neurochem. 101: 1172-1184.

Walsh DM, Klyubin I, Fadeeva JV, Cullen WK, et al. (2002). Naturally secreted oligomers of amyloid beta protein potently inhibit hippocampal long-term potentiation in vivo. Nature 416: 535-539.

Wang Y, Tang XC and Zhang HY (2012). Huperzine A alleviates synaptic deficits and modulates amyloidogenic and nonamyloidogenic pathways in APPswe/PS1dE9 transgenic mice. J. Neurosci. Res. 90: 508-517.

Yao PJ, Zhu M, Pyun EI, Brooks AI, et al. (2003). Defects in expression of genes related to synaptic vesicle trafficking in frontal cortex of Alzheimer's disease. Neurobiol. Dis.12: 97-109.

Zhang Y, Lu L, Jia J, Jia L, et al. (2014). A lifespan observation of a novel mouse model: in vivo evidence supports AB oligomer hypothesis. PLoS One 9: e85885. 